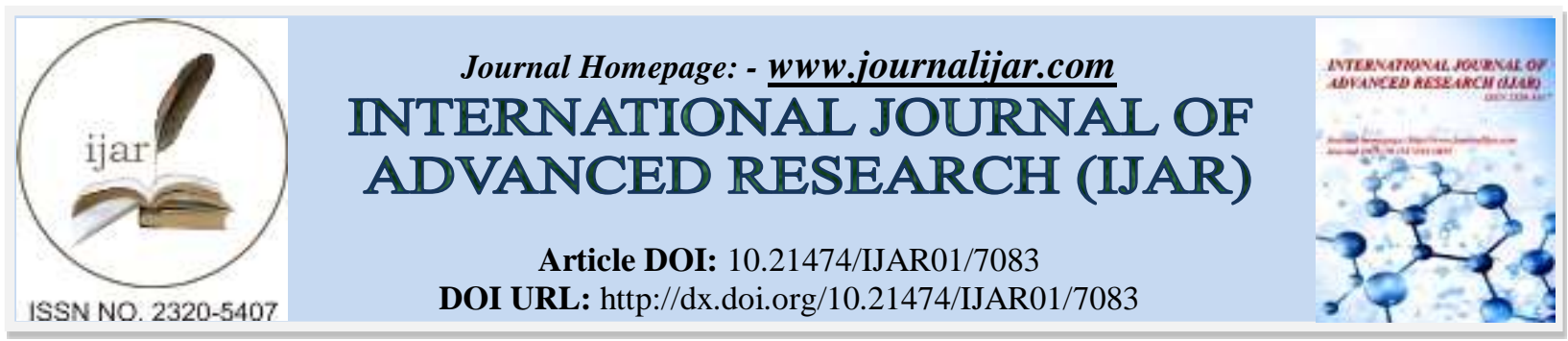

RESEARCH ARTICLE

\title{
IMPROVED UNILATERAL MIDDLE TURBINATE MUCOSAL FLAP TECHNIQUE FOR THE REPAIR OF NASAL SEPTAL PERFORATION: A SANDWICH RECONSTRUCTION BY A COMBINED USE OF UNILATERAL MIDDLE TURBINATE FLAP AND FREE INFERIOR TURBINATE GRAFT.
}

1. Okmeydanı Reserch and Education Hospital -Istanbul.

2. Yunus Emre Hospital -Istanbul.

\section{Manuscript Info}

Manuscript History

Received: 12 March 2018

Final Accepted: 14 April 2018

Published: May 2018

Keywords:-

middle turbinate, inferior turbinate, flap, graf, repair surgery, nasal septal perforation.

\section{Abstract}

Objectives: We aimed to present our experience with the improved version of previously reported unilateral middle turbinate mucosal flap technique by addition of a free graft from inferior turbinate for the repair of nasal septal perforation.

Methods: This was a retrospective series of 30 patients aged 24-45 (19 women, $11 \mathrm{men}$ ) who were operated for the reconstruction of nasal septal perforation between 2010 and 2015. Patients were evaluated clinically by anemnesis, detailed nasal examination and endoscopy. Twenty-two patients had large perforation $(2-3 \mathrm{~cm})$, while 8 had perforation $<2 \mathrm{~cm}$ in size. Sixteen patient were active cigarette smokers. The septal perforation was repaired surgically by a sandwich reconstruction technique, which includes the covering of one side of perforation by a bone containing conchal flab obtained from middle turbinate and the other side by a graft obtained from free inferior turbinate.

Results: The patients were followed up postoperatively for 20 months (18-24 months range). The nasal septal perforation was completely closed in 29 out of 30 patients, providing a success rate of $96.7 \%$. In only one patient who was actively smoking, the repair was failed.

Conclusion: Bilateral sandwich reconstruction, which is the the combined use of middle turbinate flap and free inferior turbinate graft should be considered as an alternative repair technique for large nasal septal perforations.

Copy Right, IJAR, 2018,. All rights reserved.

\section{Introduction:-}

The nasal septal perforation presents a significant reconstruction challenge depending on its etiology, location, size, and technique used for closure (Kridel, 2004; Watson and Barkdull, 2009; Moon et al., 2011). Although various surgical techniques have been suggested for the repair of nasal septal perforation in literature, none of them is reproducibly effective in clinical practice, thus search for the best technique continues (Goh and Hussain, 2007; Kim and Rhee, 2012).

The techniques in literature have suggested using local mucosal flaps alone for unilateral closure or in combination with grafts for bilateral closure. Bilateral closure technique using both mucosal flap and autogenous graft combines 
the advantages of providing migration of respiratory mucosa and rich vascular supply into the area of perforation, resulting in higher closure rates (Chhabra and Houser, 2012). In the previous studies, the closure rates ranged from $30 \%$ to $92 \%$ when using local mucosal flaps and from $67 \%$ to $100 \%$ when using both local mucosal flaps and grafts (Goh and Hussain, 2007; Li et al., 2011; Lee et al., 2008; Chhabra and Houser, 2012). Even though unilateral closure by using local mucosal flaps is a more practical technique with short operation time and acceptable outcomes for small or medium sized perforations, bilateral repair is needed to obtain effective and long-lasting closure for large perforations $(>2 \mathrm{~cm})$.

In this study, we aimed to present our experience with the improved version of our previously reported technique for the repair of nasal septal perforation (Hanci and Altun, 2014). This improved technique is a 'bilateral sandwich reconstruction' which includes the covering of one side of perforation by a flab obtained from middle turbinate and the other side by a free graft obtained from contralateral inferior turbinate. We hypothetized that bilateral closing of the nasal septal perforation with this modified technique would provide effective and durable closure for large perforations.

\section{Methods:-}

\section{Study design and population}

This was a retrospective series of 30 patients aged 24-45 (19 women, 11 men) who were operated for the reconstruction of nasal septal perforation (Figure 1) between 2010 and 2015 in Okmeydan1 Reserch and Education Hospital, ENT Clinics, Istanbul. The exclusion criteria was previously resected middle or contralateral turbinate.

The study was approved by the Institutional Ethics Committee, and the informed consent requirement was waived due to the study's retrospective design. The study was conducted according to Declaration of Helsinki.

\section{Clinical assessment:-}

Patients were evaluated clinically by anemnesis, detailed nasal examination and endoscopy. Additionally, preoperative paranasal computed tomography images were obtained for all patients. The most common preoperative symptoms were nasal obstruction $(n=22)$ and crusting $(n=20)$ followed by epistaxis $(n=15)$ and whistling $(n=6)$. The etiology of septal perforation was previous surgery in 22 patients, trauma in 3 patients, and other reasons in 6 patients. Twenty-two patients had large perforation $(2-3 \mathrm{~cm}$ ), while 8 had perforation $<2 \mathrm{~cm}$ in size (Table 1). Sixteen patient were active cigarette smokers. None of the patients had Wegener's granulomatosis. All patients take a preoperative antibiotic therapy for 14 days.

\section{Surgical technique:-}

The septal perforation was repaired surgically by a sandwich reconstruction technique, which includes the covering of one side of perforation by a bone containing conchal flab obtained from middle turbinate and the other side by a graft obtained from contralateral or ipsilateral free inferior turbinate. All the operations were performed by the same surgeon (D.H) under endoscopic view (Figure 2) while patients were under general anesthesia. The external incision was not applied in any patient. All patients received preoperative antibiotics treatment (14 days before operation), intraoperative and postoperative antibiotics.

In order to reduce intraoperative bleeding, the nasal septum, middle turbinate and contralateral inferior turbinate were injected with $1 \%$ lidocaine with 1:100,000 epinephrine solution. The nose was decongested for 5 minutes by using Xsilometazolin HCI (otrivine) on cotton nasal pledgets, after which the nasal cavities were examined and size septal perforation was measured. The mucosa around the perforation was then incised circumferentially approximately $3 \mathrm{~mm}$ from its edge. Posterior margin of the perforation was easily elevated with a blade using the tip of $20 \mathrm{G}$ needle whereas angulated instruments were needed for the elevation of mucosal flaps at the anterior, superior and inferior margins of the perforation (Figure 3). The most appropriate middle turbinate for flap and contralateral inferior turbinate for graft were chosen by considering their size, location, and computed tomography image.

The middle turbinate was lateralized and dissected from the midline starting from superior to inferior and anterior to posterior, and turbinate was opened like a leaf. The conchal bone was removed circumferentially, approximately 5 $\mathrm{mm}$ from its edge in order to obtain a monopedicled superiory based bone included conchal flap. The length of the flap was adapted to the size of the septal defect being the graft slightly larger than the perforation size. The flap was positioned between mucoperichondrial flap and septal cartilage, and then sutured to the septal mucosa of the 
anterior, posterior and inferior margin of the perforation with a reabsorbable suture (5-0 polyglycolic acid) (Figure 4).

For the repair of other side of septal perforation, a free graft slightly larger than the perforation size was prepared by dissecting contralateral or ipsilaterali inferior turbinate and plased between septal mucosa and septal structures and then sutured to the other side of septal mucosa of the anterior, posterior and inferior margin of the perforation with a reabsorbable suture (5-0 polyglycolic acid) (Figure 5). Silastic splints were subsequently placed bilaterally.

The nasal splints were removed 10 days after the operation. Saline nose drops and antibiotic cream were prescribed to keep the nose moist and clean during the postoperative 30 days period. The flap pedicle was cut by a scissor under local anesthesia 2 months after surgery, and bipolar cauterization was used to control bleeding. No intraoperative or postoperative complication was encountered.

\section{Results:-}

The patients were followed up postoperatively for 20 months (18-24 months). The assessment of the surgical outcome was based on the findings of endoscopic examination during postoperative follow-up as well as patient's symptomatology. Accordingly, the nasal septal perforation was completely closed in 29 out of 30 patients, providing a success rate of $96.7 \%$ (Figure 6). In only one patient who was actively smoking, the repair was failed.

Preoperative signs and symptoms of septal perforation like nasal obstruction, crusting, epistaxis, and whistling improved during postoperative follow-up in all patients who had successful closure of perforation. There was no finding of complication including bleeding on postoperative endoscopic examinations. The purulent postnasal drip, atrophic rhinitis symptoms, heavy crusting or foul odor was not recorded in any patient.

\section{Discussion:-}

The nasal septal perforation is a relatively common condition with a prevalence of $2-3 \%$ in general population (Simsek et al., 2014). Especially anterior and large perforations cause disturbing symptoms thus need to be repaired surgically, which is challenging procedure due to high rate of reperforation (Kridel, 2004; Watson and Barkdull, 2009; Moon et al., 2011). Bilateral coverage of moderate to large septal perforations with vascularized mucosal flap is the most important factor for complete closure and restoration of normal functionality in large perforations (Moon et al., 2011; Kim and Rhee, 2012; Park et al. 2013; Burstein and Kridel, 2013). In the previous studies, conchal cartilage, remnant septal cartilage, septal bone, temporalis fascia or inferior turbinate was used to obtain a free graft (Kilty et al., 2007; Mansour, 2011; Tastan et al., 2012; Park et al., 2013; Jeon et al., 2014). Nasal or extranasal mucosal flaps have been used previously, among which nasal flaps achieving normal nasal physiology more effectively (Watson and Barkdull, 2009; Mobley et al., 2001; Kridel, 2004). Tastan et al. (2012) used inferior turbinate composite graft alone or in combination with bipedicled mucosal advancement flap to repair medium to large nasal septal perforation in 27 patients and obtained a closure rate of 88.8\%. Mansour (2011) applied inferior turbinate free graft between the mucoperichondrium of both septum sides in 6 patients with medium size perforation and reported a closure rate of $83 \%$. Ozdek et al., (2014) used bilateral intranasal advancement/rotation flaps with open septoplasty technique in 28 patients with a success rate of $86 \%$. Islam et al., (2009) compared the efficacy of cross-stealing, advancement flap, and rotation flap techniques for the repair of nasal septal perforation in 21 patients, and recorded that the best outcome, particularly for large perforations, was obtained by the advancement or rotation flap techniques, which contain closure of perforation by both mucoperichondrial flap and interpositional graft. In the present technique, we used middle turbinate flap and contralateral free inferior turbinate graft to repair nasal septal perforation from both sides. We provided $96.7 \%$ closure rate in our series, majority of whom (22 of 30 patients) had large septal perforation $(2-3 \mathrm{~cm})$. Therefore, our success rate was even higher than the previous studies applying bilateral repair techniques (Ozdek et al., 2014; Mansour, 2011; Tastan et al., 2012).

The most remarkable advantages of the present bilateral sandwich reconstruction technique are being easy to perform through endonasal approach without any need for external incision and bringing tissues with vascular supply and mucosal structure into the area of septal perforation, which provides effective and physiological closure of large nasal septal perforations. In our previous study presenting the outcome of one-sided repair of septal perforation with middle turbinate flap, we have achieved slightly lower closure rate, which was $93.5 \%$ (Hanci and Altun, 2014). In the present report, we improved our technique by addition of a free graft from contralateral inferior turbinate and obtained even higher closure rate-96.7\%. Endonasal approach allows intraoperative assessment of 
middle and inferior turbinates as well as the area of septal perforation, ensuring precision in all steps of surgery in addition to being fast and straight forward technique. The only disadvantage of this technique over our previously reported unilateral repair technique is its requirement for longer duration of surgery due to bilateral closure which adds a second step of closure by inferior turbinate graft. It is also important to note that since the repair was bilateral, the smell function could deteriorate. However, although we did not evaluate the olfactory ability of our patients, none had complaints on the smell function during the follow-up. Indeed, in our previous study, we found that the reconstruction of nasal septal perforation with bilateral repair increased respiratory airflow and olfactory function (Altun and Hanci, 2015).

The main limitation of our study is its small sample size, which precludes us from reaching to a more definitive conclusion on the effectiveness and long-term outcome of the presented technique of sandwich reconstruction for the repair of nasal septal perforation. Still, it is one of the largest series in literature on surgical techniques for repairing nasal septal perforations. Nevertheless, our findings suggest that repairing nasal septal perforation using this technique provides high closure rate, which needs to be confirmed by large, multi-center, prospective series.

In conclusion, bilateral sandwich reconstruction, which is the combined use of middle turbinate flap and contralateral free inferior turbinate graft should be considered as an alternative repair technique for nasal septal perforations with the advantage of endonasal approach. We suggest that after careful clinical evaluation of size and location of nasal septal perforation and nasal anatomy, bilateral sandwich reconstruction technique can be applied with high closure rates for nasal septal perforations over $2 \mathrm{~cm}$.

\section{Conflicts of interest disclosure:-}

Authors declared no conflicts of interest.

Table 1:- Demographics and clinical characteristics of nasal septal perforation of 30 patients operated

\begin{tabular}{|l|l|l|}
\hline \multicolumn{2}{|l|}{ Parameters } & \multicolumn{2}{l|}{ Result } \\
\hline \multirow{2}{*}{ Age (years), mean \pm standard deviation (range) } & $32.8 \pm$ SD (24-45) \\
\hline \multirow{2}{*}{ Sender, $n(\%)$} & Female & $19(63.3 \%)$ \\
\cline { 2 - 3 } & Male & $11(36.7 \%)$ \\
\hline \multirow{3}{*}{ Preoperative symtoms, $n(\%)$} & Nasal obstruction & $16(53.3 \%)$ \\
\cline { 2 - 3 } & Crusting & $22(73.3 \%)$ \\
\cline { 2 - 3 } & Epistaxis & $20(66.7 \%)$ \\
\cline { 2 - 3 } & Whistling & $15(50.0 \%)$ \\
\cline { 2 - 3 } & Headache & $6(20.0 \%)$ \\
\cline { 2 - 3 } & Rhinorrhea & $4(13.3 \%)$ \\
\hline \multirow{5}{*}{ Size of nasal septal perforation, $n(\%)$} & Medium (<2 cm) & $5(16.7 \%)$ \\
\cline { 2 - 3 } & Large $(2-3 \mathrm{~cm})$ & $8(26.7 \%)$ \\
\hline \multirow{5}{*}{ Etiology of nasal septal perforation, $n(\%)$} & Septal surgery & $22(73.3 \%)$ \\
\cline { 2 - 3 } & Trauma & $22(73.3 \%)$ \\
\cline { 2 - 3 } & Aggressive cautery & $3(10.0 \%)$ \\
\cline { 2 - 3 } & Nose picking & $2(6.7 \%)$ \\
\cline { 2 - 3 } & Idiopathic & $2(6.7 \%)$ \\
\hline
\end{tabular}


Figure 1:- Schematic illustration of the septal perforation.

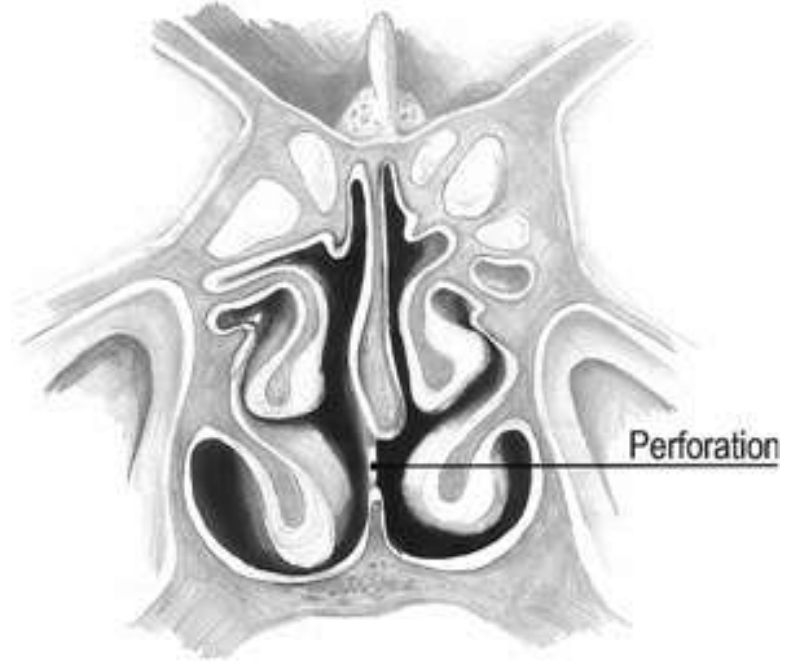

Figure 2:- Endoscopic image of the septal perforation during surgery.

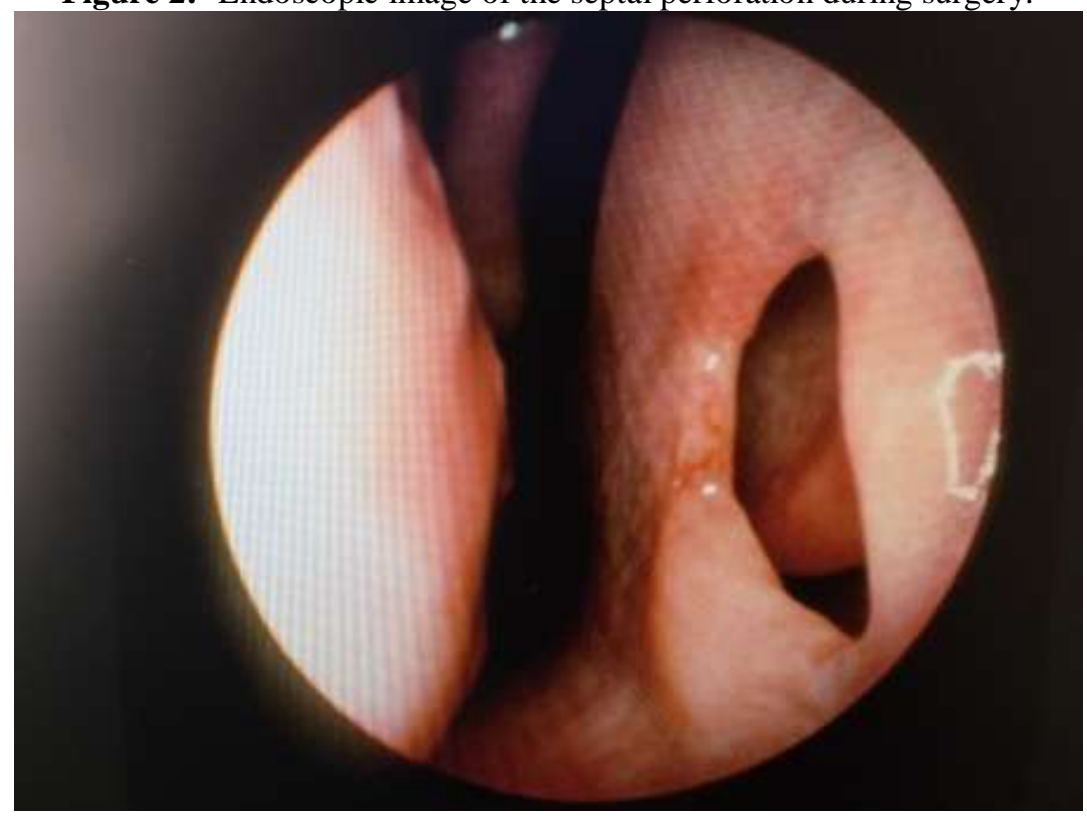


Figure 3:- Elevation of the mucosa surrounding the septal perforation by incision circumferentially approximately 3 $\mathrm{mm}$ from its edge using the angulated needles.

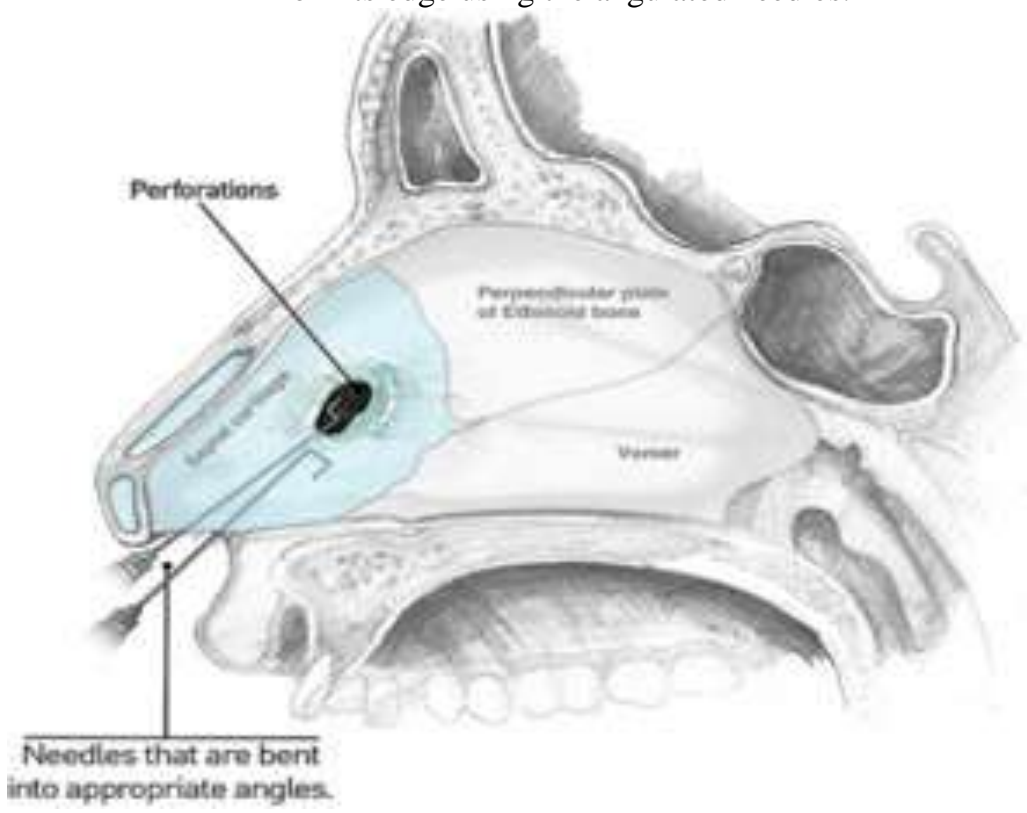

Figure 4:- Preparation of free graft from contralateral or ipsilateral inferior turbinate (a) and its fixation and stabilisation to other side of septal perforation (b).

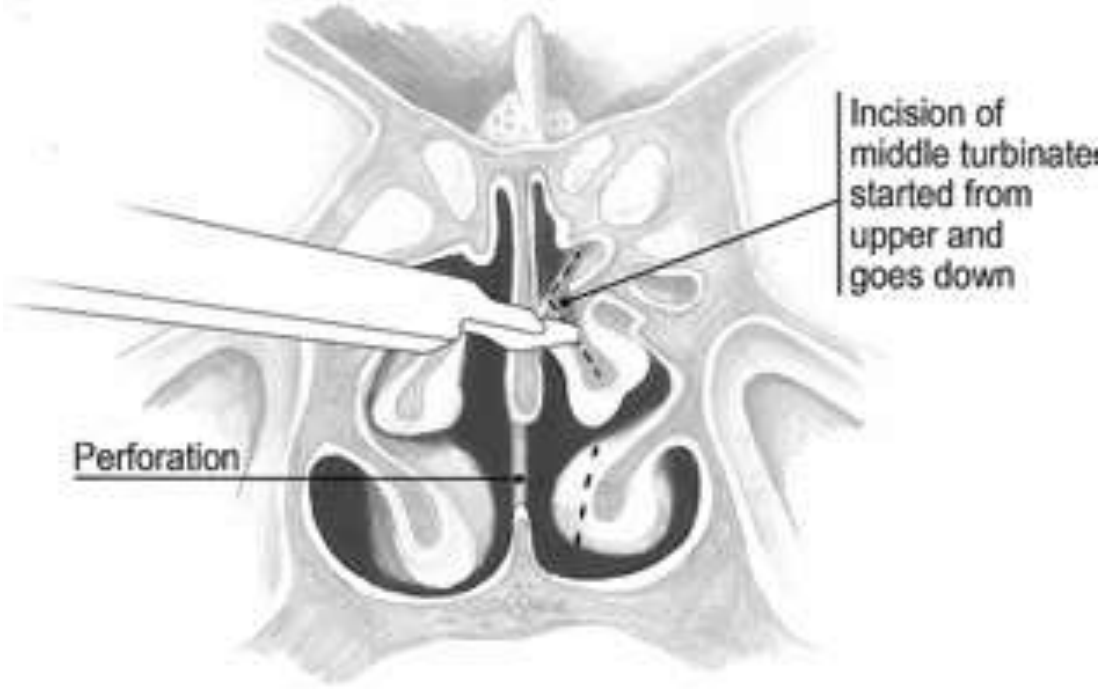




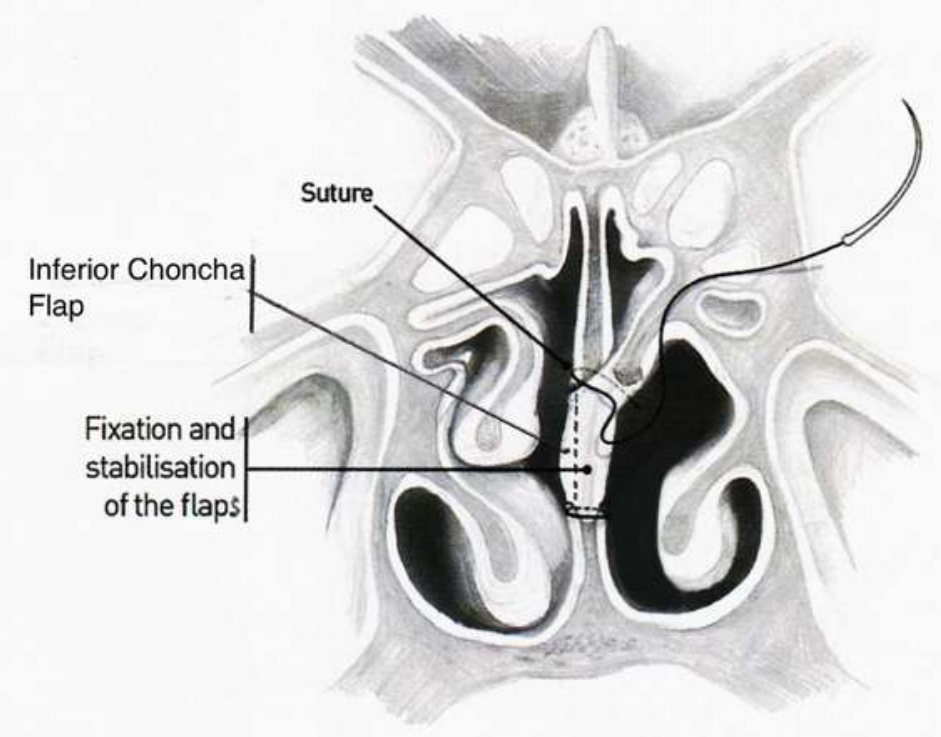

Figure 5:- Endoscopic view of repaired septal perforation from flap side (a) and from contralateral graft side (b)

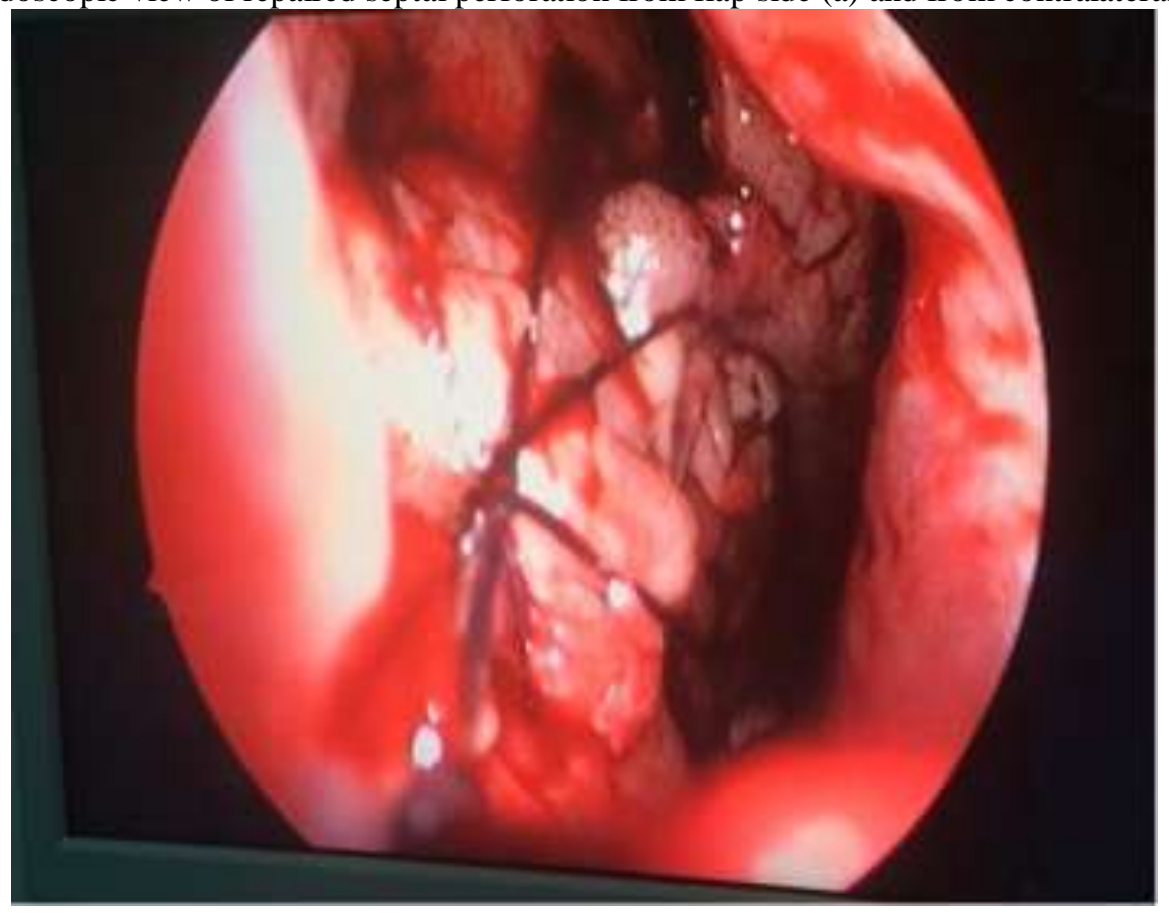




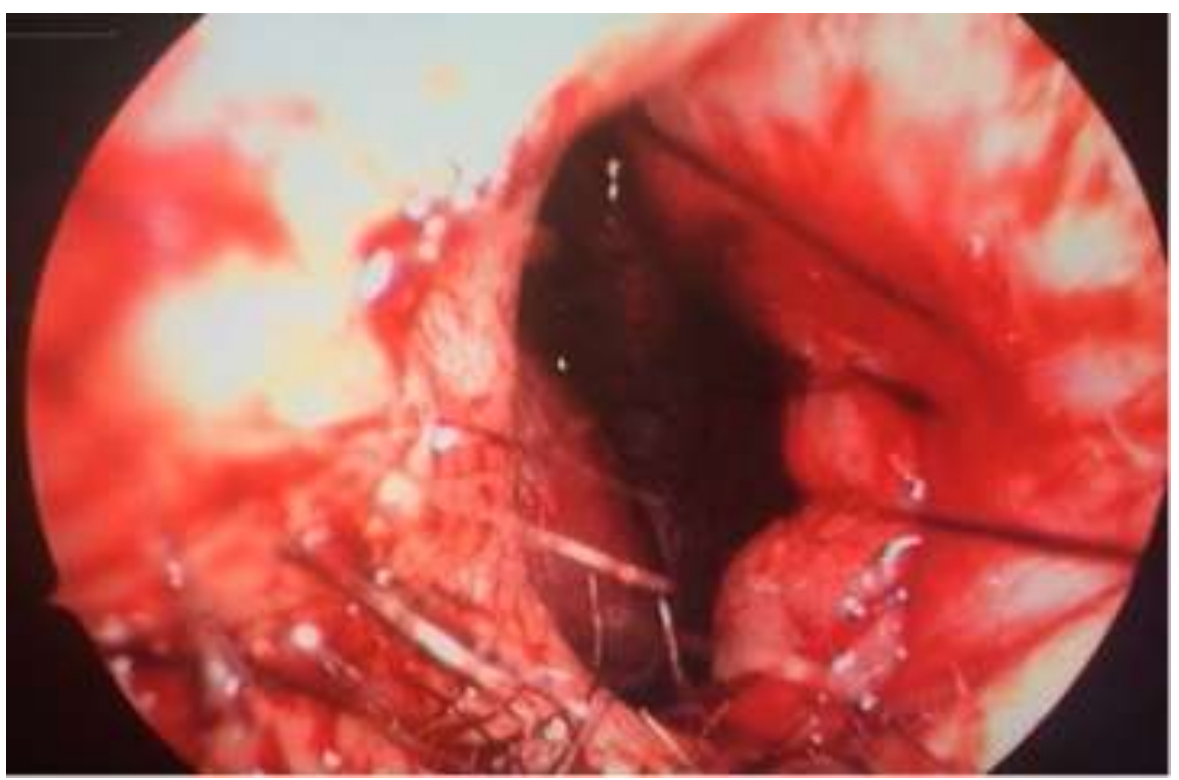

Figure 6:- Endoscopic view of the repaired septal perforation 6 months after the sandwich reconstruction surgery

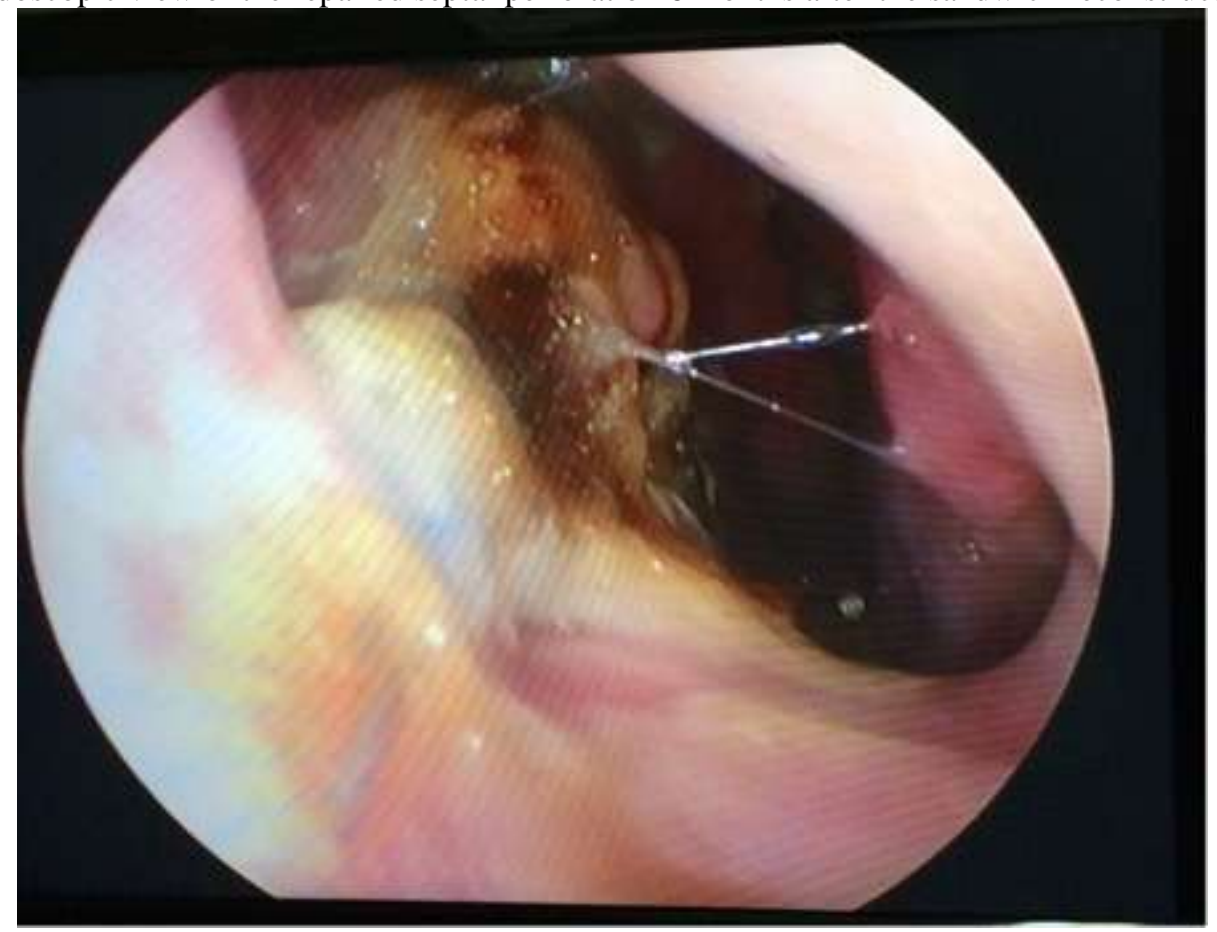




\section{References:-}

1. Altun H, Hanci D. Olfaction improvement after nasal septal perforation repair with the "cross-stealing" technique. Am J Rhinol Allergy. 2015 Sep-Oct;29(5):e142-5.

2. Burstein DH, Kridel RW. Importance of mucosal closure in nasal septal perforation repair. JAMA Facial Plast Surg. 2013 Jul-Aug;15(4):322-3.

3. Chhabra N, Houser SM. Endonasal repair of septal perforations using a rotational mucosal flap and acellular dermal interposition graft. Int Forum Allergy Rhinol. 2012 Sep-Oct;2(5):392-6.

4. Goh AY, Hussain SS. Different surgical treatments for nasal septal perforation and their outcomes. J Laryngol Otol. 2007 May;121(5):419-26.

5. Hanci D, Altun H. Repair of nasal septal perforation using middle turbinate flap (monopedicled superiory based bone included conchal flap): a new unilateral middle turbinate mucosal flap technique. Eur Arch Otorhinolaryngol. $2015 \mathrm{Jul} ; 272(7): 1707-12$.

6. Islam A, Felek S, Celik H, Arslan N, Can IH, Oğuz H. Repair of nasal septal perforation with different intranasal flap techniques and their outcomes. Kulak Burun Bogaz Ihtis Derg. 2009 Sep-Oct;19(5):232-8.

7. Jeon EJ, Choi J, Lee JH, Kim SW, Nam IC, Park YS, Jin SG, Cheon BJ. The role of temporalis fascia for free mucosal graft survival in small nasal septal perforation repair. J Craniofac Surg. 2014;25(2):e164-6.

8. Kilty SJ, Brownrigg PJ, Safar A. Nasal septal perforation repair using an inferior turbinate flap. J Otolaryngol. $2007 \mathrm{Feb} ; 36(1): 38-42$.

9. Kim SW, Rhee CS. Nasal septal perforation repair: predictive factors and systematic review of the literature. Curr Opin Otolaryngol Head Neck Surg. 2012 Feb;20(1):58-65.

10. Kridel RW. Considerations in the etiology, treatment, and repair of septal perforations. Facial Plast Surg Clin North Am. 2004 Nov;12(4):435-50.

11. Lee HR, Ahn DB, Park JH, Kim YH, Sin CM, Youn SJ, Kim JS. Endoscopic repairment of septal perforation with using a unilateral nasal mucosal flap. Clin Exp Otorhinolaryngol. 2008 Sep;1(3):154-7.

12. Li F, Liu Q, Yu H, Zhang Z. Pedicled local mucosal flap and autogenous graft for the closure of nasoseptal perforations. Acta Otolaryngol. $2011 \mathrm{Sep} ; 131(9): 983-8$.

13. Mansour HA. Repair of nasal septal perforation using inferior turbinate graft. J Laryngol Otol. 2011 May;125(5):474-8.

14. Mobley SR, Boyd JB, Astor FC. Repair of a large septal perforation with a radial forearm free flap: brief report of a case. Ear Nose Throat J. 2001 Aug;80(8):512.

15. Moon IJ, Kim SW, Han DH, Kim ST, Min YG, Lee CH, Rhee CS. Predictive factors for the outcome of nasal septal perforation repair. Auris Nasus Larynx. $2011 \mathrm{Feb} ; 38(1): 52-7$.

16. Ozdek A(1), Bayır O, Dündar Y, Tatar EC, Saylam G, Korkmaz MH. Closure of nasal septal perforations using bilateral intranasal advancement/rotation flaps. Kulak Burun Bogaz Ihtis Derg. 2014 May-Jun;24(3):123-8.

17. Park JH(1), Kim Dw, Jin HR. Nasal septal perforation repair using intranasal rotation and advancement flaps. Am J Rhinol Allergy. 2013 Mar-Apr;27(2):e42-7.

18. Simsek G. Septal perforation repair. Turkiye Klinikleri J E.N.T.-Special Topics 2014;7(2):67-79.

19. Tastan E, Aydogan F, Aydin E, Can IH, Demirci M, Uzunkulaoglu H, Unlu I. Inferior turbinate composite graft for repair of nasal septal perforation. Am J Rhinol Allergy. 2012 May-Jun;26(3):237-42.

20. Watson D, Barkdull G. Surgical management of the septal perforation. Otolaryngol Clin North Am. 2009 Jun;42(3):483-93. 